\title{
A Cyclostationarity Analysis Applied to Scaled Images
}

\author{
Babak Mahdian and Stanislav Saic \\ Institute of Information Theory and Automation of the AS CR \\ Pod Vodárenskou věží 4, 18208 Prague 8, Czech Republic \\ \{mahdian, ssaic\}@utia.cas.cz \\ http://zoi.utia.cas.cz/image-forensics
}

\begin{abstract}
The knowledge of image's geometric history plays an important role in image signal compression, image registration, image retrieval and especially in image forensics. In this paper we focus on scaling and show that images that have undergone scaling contain hidden cyclostationary features. This makes possible employing the well-developed theory and efficient methods of cyclostationarity for a blind analyzing of the history of images in respect to scaling transformation. To verify this, we also propose a cyclostationarity detection method applied to our problem and show how the traces of scaling can be detected and the specific parameters of the transformation estimated. The method is based on the fact that a cyclostationary signal has a frequency spectrum correlated with a shifted version of itself. A quantitative measure of the efficiency of the method is proposed as well.
\end{abstract}

Keywords: Interpolation; scaling; cyclostationary; authentication; image forensics.

\section{Introduction}

The knowledge of image's geometric history plays an important role in image signal compression, image registration, medical image analysis, image retrieval, digital publishing, etc. Furthermore, it has a crucial role in image forensics. Here the detection of traces of geometrical transformations signifies photo tampering (for example, see [106]). Moreover, when applying a statistical-based method to an image, without knowing the processing history of this image and how the statistics of the image has been changed, we can expect miscalculations and unexpected results. The core of the paper is based on the cyclostationarity theory, which is an attractive and novel one for the computer vision and pattern recognition community.

The term cyclostationarity refers to a special class of signals which exhibit periodicity in their statistics. In this paper, we focus on scaling and show that images that have undergone such a transformation contain hidden cyclostationary features. This will justify employing the well-developed theory of cyclostationarity and its efficient methods for analyzing images' history in respect to geometrical 
transformations. The cyclostationarity is brought into the signal by the interpolation process (nearest neighbor, linear, cubic, etc.). The interpolation process is present in almost every image resizing operation. Interpolation has a long history and probably started being used as early as 2000BC by ancient Babylonian mathematician: 1. Despite this long history, the massive usage of interpolation and its importance in digital signal processing, to our knowledge, there exist only a few published works concerned with the specific changes brought into the signal by this process [1075]. The knowledge that interpolated/resampled images are defacto cyclostationary signals makes possible a new point of view to such images and justifies employing existing efficient cyclostationarity detectors to improve the results of mentioned methods.

One of the most important properties of a cyclostationary signal is the existence of specific correlation between its spectral components [3. Based on this knowledge, we also propose a cyclostationarity detection method capable of determining whether a given digital image is result of scaling. If so, the method also can determine the specific parameters of transformation.

Comparing the method described in this paper with [10] shows, that the latter one is based on a complex and time-consuming EM estimation. Our method uses a simple and fast method for detecting cyclostationary features and achieves very similar results. Furthermore, the output of the method in [10] and the convergency of their EM part directly depend on several initialization parameters. Our method does not need any parameters initialization and work in a complete blind way.

\section{Cyclostationarity}

In the last half a century a lot of work has been done in the field of cylcostationarity 4]. Much of the initial work introducing and examining the use of cyclostationary models in the signal analysis was carried out by W. A. Gardner et al. [2113].

A zero-mean signal $f(x)$ is defined to be second order cyclostationary if its second order statistics are periodic. The autocorrelation function of $f(x)$ can be defined as

$$
R_{f}(x, \delta)=E\left\{f(x) f^{*}(x+\delta)\right\},
$$

because of its periodicity in $x$, we can represent it in the form of a Fourier series expansion:

$$
R_{f}(x, \delta)=\sum_{\alpha} R_{f}^{\alpha}(\delta) e^{j 2 \pi \alpha x},
$$

where $\alpha$ is the cyclic frequency. The parameter $R_{f}^{\alpha}$ is called Cyclic Autocorrelation Function (CAF) and it is a fundamental parameter of cyclostationarity.

\footnotetext{
${ }^{1}$ For instance, it had an important role in astronomy which in those days was all about time-keeping and making predictions concerning astronomical events 8 .
} 
CAF is defined as:

$$
R_{f}^{\alpha}(\delta)=\lim _{X \rightarrow \infty} \frac{1}{X} \int_{-X / 2}^{X / 2} R_{f}(x, \delta) e^{-j 2 \pi \alpha x} d x
$$

An appropriate way of analyzing cyclostationary properties is by applying the Fourier Transform (FT) to $R_{f}^{\alpha}$. The result is called Spectral Correlation Function $(\mathrm{SCF})$ and is defined as:

$$
S_{f}^{\alpha}(u)=\int_{-\infty}^{\infty} R_{f}^{\alpha}(\delta) e^{-j 2 \pi u \delta} d \delta
$$

As we will deal with discrete signals, the discrete version of CAF should also be defined here:

$$
R_{f}^{\alpha}(l)=\lim _{N \rightarrow \infty} \frac{1}{N} \sum_{m=0}^{N-1} f[m] f^{*}[m+l] e^{-j 2 \pi \alpha m \Delta m},
$$

where $N$ and $\Delta m$ denote the number of samples of the signal and sampling interval, respectively. Equivalently, the discrete SCF can be obtained by:

$$
S_{f}^{\alpha}(u)=\sum_{l=-\infty}^{\infty} R_{f}^{\alpha}(l) e^{-j 2 \pi u l \Delta l}
$$

$\mathrm{CAF}$ and SCF are analogous to the autocorrelation function and power spectral density function for stationary signals. When $\alpha=0$, the SCF can also be interpreted as power spectral density of the signal. For other values of $\alpha$, SCF is cross-spectral density between the signal and the signal shifted in frequency by $\alpha$. So, if the signal being analyzed exhibits cyclostationarity, SCF will be non-zero for some $\alpha \neq 0$. Otherwise, only for $\alpha=0$ we will have non-zero values.

\section{Detecting Cyclostationarity in Scaled Images}

We will assume the following simple, linear and stochastic model and assumptions:

$$
f(x)=(u * h)(x)+n(x)
$$

where $f, u, h, *$, and $n$ are the measured image, original image, system PSF, convolution operator, and random variable representing the influence of noise sources statistically independent from the signal part of the image $(E\{n(x)\}=$ $0)$. If we consider the first part of equation (7) to be deterministic, the covariance of equation (7) can be shown to be $R_{f}\left(x_{1}, x_{2}\right)=\operatorname{Cov}\left\{f\left(x_{1}\right), f\left(x_{2}\right)\right\}=$ $E\left\{\left(f\left(x_{1}\right)-\bar{f}\left(x_{1}\right)\right)\left(f\left(x_{2}\right)-\bar{f}\left(x_{2}\right)\right)\right\}=\operatorname{Cov}\left\{n\left(x_{1}\right), n\left(x_{2}\right)\right\}=R_{n}\left(x_{1}, x_{2}\right)$, where $R_{f}$ is the covariance matrix of measured image $f(x)$, and $R_{n}$ is the covariance of random process $n(x)$. 
By $f_{k}$ we will denote a discrete signal representing the samples of $f(x)$ at the locations $k \Delta, f_{k}=f(k \Delta)$, where $\Delta \in \mathcal{R}^{+}$, is the sampling step and $k \in \mathcal{N}_{0}$.

There are two basic steps in scaling transformation. In the first step a spatial transformation of the physical rearrangement of pixels in the image is done. Coordinate transformation is described by a transformation function, $T$, which maps the coordinates of the $x^{\prime}=T_{x}(x, y), y^{\prime}=T_{y}(x, y)$.

The second step is the interpolation step. Here pixels intensity values of the transformed image are assigned using a constructed low-pass interpolation filter, $w$. As the word interpolation signifies 2 , the interpolation process can be described using the following convolution:

$$
f^{w}(x)=\sum_{k=-\infty}^{\infty} f_{k} w\left(\frac{x}{\Delta}-k\right)
$$

Many interpolation kernels have been investigated and proposed so far [9]. We will be concerned with following low-order piecewise local polynomials: nearestneighbor, linear, cubic and truncated sinc. These polynomials are used extensively because of their simplicity and implementation unassuming properties. As will be shown, these interpolators bring noticeable periodic artifacts into the signal.

As was shown in [117], when considering the stochastic model and conditions stated in the beginning of this section, the variance of an interpolated signal becomes:

$$
\operatorname{var}\left\{f^{w}(x)\right\}=\operatorname{var}\left\{f^{w}(x+\vartheta \Delta)\right\}, \vartheta \in \mathcal{Z}
$$

In other words, $\operatorname{var}\left\{f^{w}(x)\right\}$ is periodic over $\mathrm{x}$ with period $\Delta$. Thus, interpolated signals contain periodically varying properties.

Now, we can employ the theory of cyclostationarity for finding the traces of scaling. Many efficient methods capable of detecting cyclostationary features 4[12 have been proposed so far.

Theory of cyclostationarity has shown that a cyclostationary signal has a frequency spectrum that is correlated with a shifted version of itself [1]. Based on this, we on focus detecting the traces of cyclostationarity by estimating the spectral correlation function. To estimate the SCF, we can simply use equation (6). But, due to its computational complexity, instead of this, we rather use a more computationally effective SCF estimation method based on Fast Fourier Transform (FFT). FFT algorithm has computational complexity $\mathrm{O}\left(\mathrm{nlog}_{2} \mathrm{n}\right)$.

Let's say $f(x, y)$ is the image being analyzed and $F(n, u)$ is a matrix containing FFT of image's rows (i.e., $F(1, u)$ contains the one-dimensional FFT of the first row of $f(x, y))$. The SCF can be estimated in the following way:

$$
S_{f}^{\alpha}(u)=\frac{1}{N} \sum_{n=0}^{N-1} F_{n, u} \cdot F_{n, u+\alpha}^{*},
$$

\footnotetext{
$\overline{2}$ The word "interpolation" originates from the Latin word "inter", meaning "between", and verb "polare", meaning "to polish" 8 .
} 
(a)

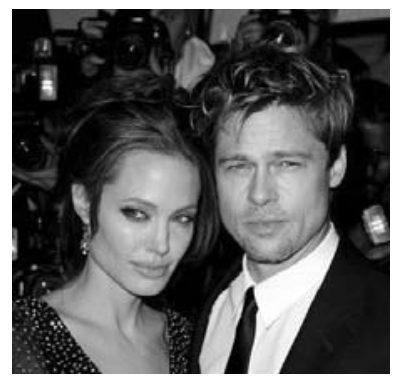

(d)

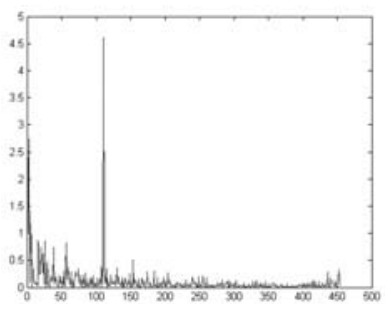

(b)

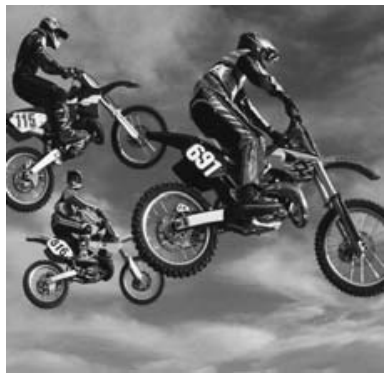

(e)

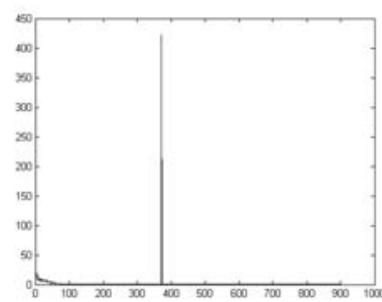

(c)

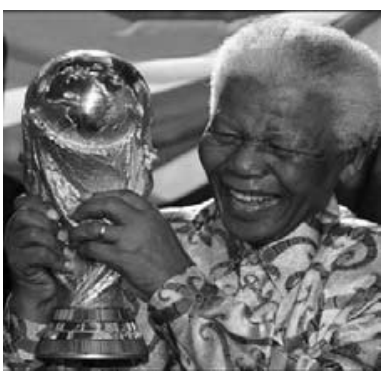

(f)

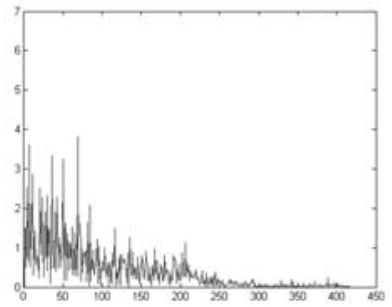

Fig. 1. Each column shows an example image and a corresponding correlation map obtained by application of equation (11) to the resized version of this image. The used scaling rates were (from left to right) 1.32. 1.7 and 1.2. In other words, Figure 1(e) was obtained by applying the method to resized version (with scaling rate 1.7) of Figure 1 (b). In all cases the bicubic interpolation has been used. The distinctive peaks signifying the resizing procedure.

where $*$ denotes complex conjugate and $N$ is the number of image's rows.

Data obtained can be combined together to create the resulting correlation map:

$$
\rho_{f}(\alpha)=\sum_{u}\left|S_{f}^{\alpha}(u)\right|^{2}
$$

To demonstrate the method's output obtained using equation (11) we apply it to several images resized by various scaling factors, see Figure 1. Here, to get clear peaks, columns of $F(n, u)$ were scaled to have values between 0 and 1 . As apparent from Figure 1 cyclostationary features resulting from the scaling procedure are exhibited by distinctive peaks.

\subsection{Adaptation of the Proposed Method}

The method presented so far works well (produces clear peaks) when the scaling rate is big enough to introduce strong correlation into the image. When the image is transformed by a lower scaling rate, the cyclostationary features are not strong enough to be detectable using the basic method (see Figure 1 (c)). This drawback can be overcome using a traditional way based on passing the 
(a)

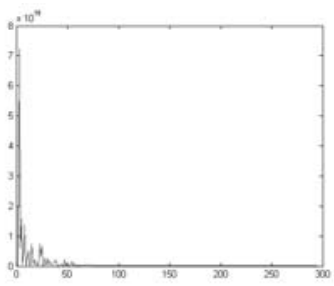

(d)

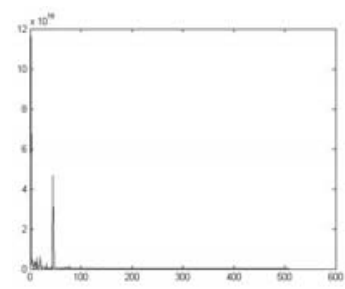

(b)

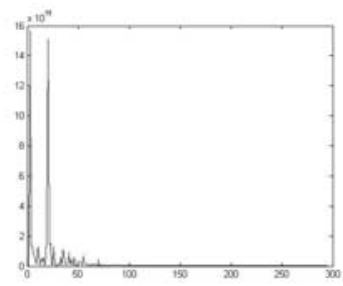

(e)

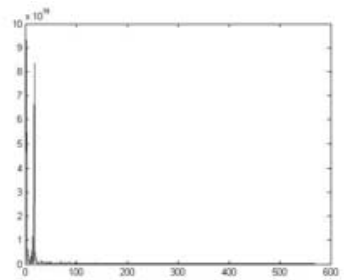

(c)

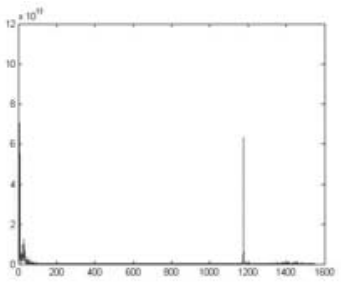

(f)

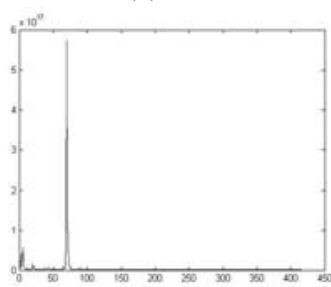

Fig. 2. A few examples of the method's output. In (a) the output of the image applied to the non-resized version of Figure 1(a) is shown. As apparent, there is no clear peak. (b) and (c) show the output of the method for resized Figure 1 (a) with scaling rates 1.07 and 4.11 respectively. (d) and (e) show the output of the method for resized Figure 1 (b) with scaling rates 0.92 and 1.03 . In (e) the output of the method applied to the resampled version of Figure 1(c) with scaling rate 1.2 is shown. In all cases the bicubic interpolation has been used.

analyzed image through a set of band-pass filters. We use a set of derivative filters as band-pass filters. If $f(x, y)$ denotes the image being analyzed, $\mathbf{d}_{\mathbf{x}}$ and $\mathbf{d}_{\mathbf{y}}$ are band-passed images containing the horizontal and vertical derivative approximations:

$$
\mathbf{d}_{\mathbf{x}}=\left[\begin{array}{ll}
-1 & 1
\end{array}\right] * f(x, y) \quad \mathbf{d}_{\mathbf{y}}=\left[\begin{array}{c}
-1 \\
1
\end{array}\right] * f(x, y)
$$

Applying the equations (10) and (11) to $\mathbf{d}^{\mathbf{n}}$,

$$
\mathbf{d}^{\mathbf{n}}=\mathbf{d}_{\mathbf{x}}^{\mathbf{n}}+\mathbf{d}_{\mathbf{y}}^{\mathbf{n}},
$$

where $\mathbf{n}$ denotes the order of derivative filter, results in significantly more accurate and robust outcomes. After performing various experiments with resized images of different structures, brightness and noise characteristics we achieved good results using only a lower and a higher derivative filter. The proposed method consists of application of equation (10) to $\mathbf{d}^{\mathbf{1}}$ and $\mathbf{d}^{\mathbf{5}}$ separately:

$$
\begin{aligned}
& \rho_{\mathbf{d}^{1}}(\alpha)=\sum_{u}\left|S_{\mathbf{d}^{1}}^{\alpha}(u)\right|^{2} \\
& \rho_{\mathbf{d}^{5}}(\alpha)=\sum_{u}\left|S_{\mathbf{d}^{5}}^{\alpha}(u)\right|^{2}
\end{aligned}
$$


If the image being analyzed has been scaled, then at least one of the correlation maps $\rho_{\mathbf{d}^{1}}(\alpha), \rho_{\mathbf{d}^{5}}(\alpha)$ will exhibit a detectable peak (see Figure2). In all examples, the method has been applied to the green color band.

Detected peaks are directly related with the scaling rate. So, using the position of the occurred peak, the particular scaling rate can be estimated. For example, when assuming the upsampling operation and $a$ will denote the position of the peak in interest obtained by applying the method to $f(x, y)$, then the scaling rate can be estimated in the following way: $s=\frac{y}{y-a}$.

\section{Quantitative Experiments}

The method has been applied to 1000 images undergone various scaling transformations. The size of the investigated images was $512 \times 512$ pixels. All experiments were carried out in Matlab. In all cases never-compressed images have been used. Table 1 shows the detection accuracy of the method applied to bicubic resized images. The detection accuracy expresses the success of the method in expressing the interpolation by a clear and easily detectable peak either in $\rho_{\mathbf{d}^{1}}(\alpha)$ or $\rho_{\mathbf{d}^{5}}(\alpha)$. Note that the detection is nearly perfect for scaling rates greater than 0.90 . Here, the amount of the cyclostationary features is strong enough to be detectable. When the image is downsampled, the power of cyclostationary features brought into the signal is weakened and a lot of information is lost (due to downscaling). This makes the detection of downsampling difficult. Shown statistics for scaling rate 1 (non-resized) correspond to the false positives rate of the method. Here, if no peaks are found, the image is denoted as non-resized.

Table 1. Detection accuracy [\%]. Each cell corresponds to the average detection accuracy from 1000 images.

\begin{tabular}{|c||c|c|c|c|c|c|c|c|c|c|}
\hline scaling rate & 0.75 & 0.80 & 0.85 & 0.90 & 0.95 & 0.97 & 1.00 & 1.03 & 1.05 & 1.10 \\
\hline accuracy & 24 & 91 & 93 & 98 & 100 & 100 & 95 & 100 & 100 & 100 \\
\hline scaling rate & 1.20 & 1.40 & 1.50 & 1.60 & 1.70 & 1.80 & 1.90 & 2.00 & 2.10 & 2.20 \\
\hline accuracy rate & 100 & 100 & 100 & 100 & 100 & 100 & 100 & 100 & 100 & 100 \\
\hline
\end{tabular}

\section{Conclusions and Further Research}

The aim of this paper was to show that images that have undergone a geometric transforation contain hidden cyclostationary features. This makes possible employing the existing efficient cyclostationary detectors to find the traces of such transformations. In order to verify this, we focused on scaling and carried out several experiments based on a common cyclostationarity detector. Results obtained are promising and show that employing cyclostationarity methods can be effective. Further research might explore application and evaluation of various types of cyclostationary feature detection methods, use of filter banks and extension to other geometric transformations, such as rotation and arbitrary affine transformations. 
Acknowledgments. This work has been supported by the Czech Science Foundation under the project No. GACR 102/08/0470.

\section{References}

1. Gardner, W.A.: The spectral correlation theory of cyclostationary time-series. Signal Process 11(1), 13-36 (1986)

2. Gardner, W.A.: Spectral correlation of modulated signals: Part i-analog modulation. IEEE Transactions on Communications 35(6), 584-594 (1987)

3. Gardner, W.A.: Exploitation of spectral redundancy in cyclostationary signals. IEEE Signal Processing Magazine 8(2), 14-36 (1991)

4. Gardner, W.A., Napolitano, A., Paura, L.: Cyclostationarity: Half a century of research. Signal Processing 86(4), 639-697 (2006)

5. Kirchner, M.: Fast and reliable resampling detection by spectral analysis of fixed linear predictor residue. In: Proceedings of the 10th ACM workshop on Multimedia and security, pp. 11-20. ACM Press, New York (2008)

6. Mahdian, B., Saic, S.: Detection of copy-move forgery using a method based on blur moment invariants. Forensic science international 171(2-3), 180-189 (2007)

7. Mahdian, B., Saic, S.: Blind authentication using periodic properties of interpolation. IEEE Transactions on Information Forensics and Security 3(3), 529-538 (2008)

8. Meijering, E.: A chronology of interpolation: From ancient astronomy to modern signal and image processing. Proceedings of the IEEE 90(3), 319-342 (2002)

9. Meijering, E.H.W., Niessen, W.J., Viergever, M.A.: Piecewise polynomial kernels for image interpolation: A generalization of cubic convolution. In: ICIP (3), pp. 647-651 (1999)

10. Popescu, A., Farid, H.: Exposing digital forgeries by detecting traces of re-sampling. IEEE Transactions on Signal Processing 53(2), 758-767 (2005)

11. Rohde, G., Berenstein, C., Healy, D.: Measuring image similarity in the presence of noise. In: Proceedings of the SPIE Medical Imaging: Image Processing, February 2005, vol. 5747, pp. 132-143 (2005)

12. Serpedin, E., Panduru, F., Sari, I., Giannakis, G.B.: Bibliography on cyclostationarity. Signal Process 85(12), 2233-2303 (2005) 